\title{
Multiparameter Stability Analysis of Systems with Induction Motor Loads, Weak Interconnections and Series Compensation
}

\author{
JONATHAN DEVADASON, PAUL S. MOSES \\ University of Oklahoma \\ Electrical and Computer Engineering \\ 110 W.Boyd St, Norman OK 73019
}

USA

\author{
MOHAMMAD A.S. MASOUM \\ Utah Valley University \\ Department of Engineering
}

800 West University Parkway, Orem UT 84058

USA

Abstract: Dynamic modeling and stability domain analysis of a system consisting of a synchronous generator supplying an induction motor load through a series compensated weak network has been carried out in this paper. The impact of $X / R$ ratio of the feeder and generation control system parameters on the stability domain with respect to series compensation has been examined through eigenvalue calculations and time domain simulations. From the studies conducted, it was observed that the stability domain of the system with respect to series compensation depends on the grid strength in addition to the excitation system parameters. Eigenvalue analysis shows that there is a strong correlation between the exciter gain, time constants of the measurement transducer and exciter, and the series compensation level. The main contribution of this work is to reveal new bifurcations which arise in these systems which has been studied through eigenvalue analysis and time domain simulations for various combinations ofsystemparameters.

Key-Words: stability, series compensation, eigenvalues, subsynchronous resonance, bifurcations, synchronous generators, inductionmotors

Received: December 2, 2020. Revised: May 17, 2021. Accepted: June 7, 2021. Published: June 26, 2021.

\section{Introduction}

Utilizing series capacitors is an inexpensive way of improving the power transfer capability of the existing transmission infrastructure while simultaneously improving the short circuit capacity and thereby increasing grid strength. However, one of the well-known factors limiting the amount of series compensation is subsynchronous resonance (SSR) which has known to cause excessive voltages and currents due to induction generator effect and torsional oscillations as a result of the interaction between the mechanical system of the synchronous machine and the series compensated network ([1], [2], [3], [4]).

The influence of large dynamic loads on power system stability has been studied in [5]. Although the exciter gain and direct axis time constant of the induction motor has been found to impact stability, the influence of time constants of the measurement transducer and the exciter has not been modeled. In this paper, it is shown that the time constants of the exciter and measurement transducer has a strong influence on the stability of the system especially when a series compensated network is considered.

Traditionally, studies on SSR are performed on systems consisting of a generator or a set of generators feeding power to an infinite bus through a series compensated network by applying bifurcation theory to the IEEE First Benchmark Model ([6], [7], [8], [9]).
Bifurcation theory has been applied to study SSR in the IEEE Second Benchmark Model in [10]) where the influence on exciter gain on SSR has been analyzed. However, the time constant of the exciter is assumed to be fixed, and its influence on SSR has not been addressed. Global bifurcations have been studied in the IEEE First Benchmark Model due to variations in damping and mechanical power of the synchronous generator ([11], [12]). Also, ferroresonance in single and three-phase transformers has been investigated using bifurcation theory ([13], [14]). Few studies have also been performed on induction motor loads fed through a series compensated feeder from a substation which is modeled as an infinite bus ([15], [16], [17]).

In all the references stated so far, there is an assumption of a stiff voltage source namely the infinite bus which maybe equivalent to a substation in real time. However, the impact of generator voltage controller parameters on loads supplied from the substation has not been studied so far. Hence, the goal of this work is to analyze the impact of the generation system on induction motor load operation when the network is series compensated. This work is important because existing transmission systems are getting weaker due to increased distance between generation and load centers resulting in power being transmitted through long feeders of high impedance which 


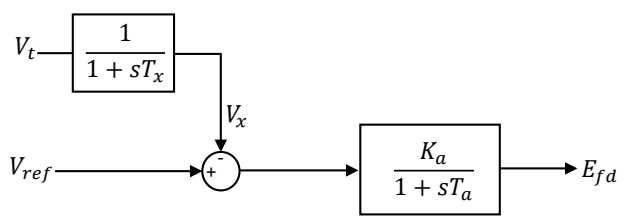

Figure 2: Block diagram of AVR control system

makes series compensation an economical and simple method to increase power flow through the system. The applications of this work would help identify the cause of instability in a series compensated network as instability maybe triggered due to exciter system crossing certain bounds depending on the series compensation level in addition to SSR.

This paper is organized as follows: the system under study and eigenvalue analysis of the system for various combinations of parameters are presented in Sections 2 and 3 respectively. Section 4 describes time domain simulation results for some combinations of system parameters. Finally, conclusions drawn from the studies conducted are summarized in Section 5.

\section{System under study}

The single line diagram of the system under study is shown in Fig. 1. It consists of a synchronous generator (SG) supplying power to a substation (SS) through a transformer and a short line. The substation is connected to an induction motor load through a series compensated feeder.

The synchronous machine is represented using 4 differential equations which describe $d$ and $q$ axis components of the stator currents $\left(I_{d}\right.$ and $\left.I_{q}\right)$ and the induced emfs in the field $\left(E_{q}^{\prime}\right)$ and damper winding $\left(E_{d}^{\prime}\right)$ on the rotor. The stator of the synchronous machine is directly connected to the network which means the stator currents of the synchronous machine are the same as the network currents. Electromechanical transients of the synchronous generator are expressed using 2 differential equations which describe the rotor speed $(\omega)$ and the rotor position $(\delta)$. The detailed dynamic model of the synchronous machine can be found in [18].

The electromagnetic transients of the induction motor are represented using 4 differential equations which govern the behavior of $d$ and $q$ components of stator $\left(I_{d s}, I_{q s}\right)$ and rotor currents $\left(I_{d r}, I_{q r}\right)$. Electromechanical transients are computed using the swing equation which determines the slip $\left(s_{m}\right)$ of the induction motor. The detailed dynamic model is based on [19].

The block diagram of the Automatic Voltage Regulator (AVR) of the SG is shown in Fig. 2. The terminal voltage magnitude $\left(V_{t}\right)$ of the SG is measured by a transducer of time constant $T_{x}$ seconds and is compared with the reference voltage $V_{\text {ref }}$. The AVR is represented using a first order transfer function consisting of a gain $K_{a}$ and time constant $T_{a}$ seconds. The speed governor dynamics are modeled using a first order differential equation which determines the mechanical torque input $\left(T_{m}\right)$ to the rotor of the SG. The differential equations which govern the dynamics of the AVR and the speed governor are given below:

$$
\begin{aligned}
T_{x} \dot{V}_{x} & =-V_{x}+V_{t} \\
T_{a} \dot{E}_{f d} & =-E_{f d}+K_{a}\left(V_{r e f}-V_{x}\right) \\
T_{g} \dot{T}_{m} & =-T_{m}+P_{c}-\left(1 / R_{d}\right)(\omega-1)
\end{aligned}
$$

In the above equations (1) to (3), $V_{x}$ is the output of the measurement transducer described previously, $E_{f d}$ is the input voltage applied to the field winding on the rotor of the $\mathrm{SG}, P_{c}$ is the control input which is fixed and $R_{d}$ is the droop of the speed governor. The values of $T_{g}$ and $R_{d}$ are assumed to be $50 \mathrm{~ms}$ and $5 \%$ respectively.

\section{Eigenvalue analysis}

The state-space model of the system is developed for the system and is linearized and cast in the form $\Delta \dot{X}=[J] \Delta X$ where $X$ is the vector of system state variables and $\Delta X$ is the linearized state variable vector. The state variables considered in this system are the $d$ and $q$ axis components of stator and rotor currents, terminal voltage and slip of the induction motor, field and damper winding emfs, mechanical torque, rotor angle and speed of the synchronous generator, $d$ and $q$ axis components of the line current and series capacitor voltage and variables $V_{x}$ and $E_{f d}$ associated with the AVR as explained in the previous section. The eigenvalues of the linearized mathematical model of the system are calculated and analyzed for various values of system parameters and the results are presented in the subsections that follow: 
Table 1: Parameters of synchronous generator

\begin{tabular}{|c|c|c|c|c|c|c|c|}
\hline$H_{g}$ & $R_{a}$ & $X_{d}$ & $X_{q}$ & $X_{d}^{\prime}$ & $X_{q}^{\prime}$ & $T_{d o}^{\prime}$ & $T_{q o}^{\prime}$ \\
\hline $4 \mathrm{~s}$ & $0.001 \mathrm{pu}$ & $1.75 \mathrm{pu}$ & $1.65 \mathrm{pu}$ & $0.3 \mathrm{pu}$ & $0.75 \mathrm{pu}$ & $5 \mathrm{~s}$ & $1 \mathrm{~s}$ \\
\hline
\end{tabular}

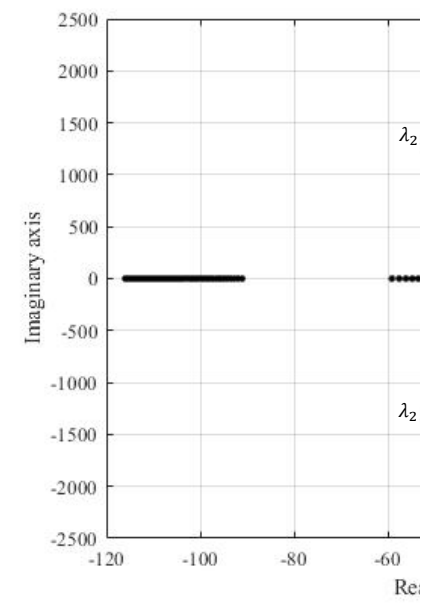

Figure 3: Eigenvalue trc

\subsection{Eigenvalues of bast}

In this section, eigenvalues of the system are computed for the following operating conditions: the induction motor slip is at $2 \%$ and the $X / R$ ratio of the line is assumed to be 20, exciter of the SG has a gain and time constant of 500 and $25 \mathrm{~ms}$ respectively and the measurement transducer time constant of the AVR is $10 \mathrm{~ms}$. The series compensation level of the system $\left(K_{c}=X_{C} / X_{n e t}, X_{n e t}=X_{T 1}+X_{L 1}+X_{T 2}+X_{L 2}+\right.$ $\left.X_{T 3}\right)$ is varied from $1 \%$ to $95 \%$.

The eigenvalues of the system along with the corresponding dominant state variables for each mode are presented in Table 3. for a series compensation of $60 \%$. There are 6 oscillatory modes $\left(\lambda_{1}\right.$ to $\left.\lambda_{6}\right)$ and 5 non-oscillatory modes $\left(\lambda_{7}\right.$ to $\left.\lambda_{11}\right)$. The system starts out to be stable as the series compensation is at $1 \%$ and remains stable up to $62 \%$. As the compensation is increased beyond $62 \%$, the system becomes unstable due to SSR as the oscillatory mode $\lambda_{4}$ crosses over to the right half of the complex plane resulting in an unstable system. The same analysis when repeated for a lower $X / R$ ratio of the system $(X / R=10)$ revealed a Hopf bifurcation due to SSR at a higher value of critical series compensation $\left(K_{c r}=74 \%\right.$, $\left.\lambda_{c r}=0.0522 \pm j 192.07\right)$ and for a higher $X / R$ ratio of 30 , the critical value of series compensation is

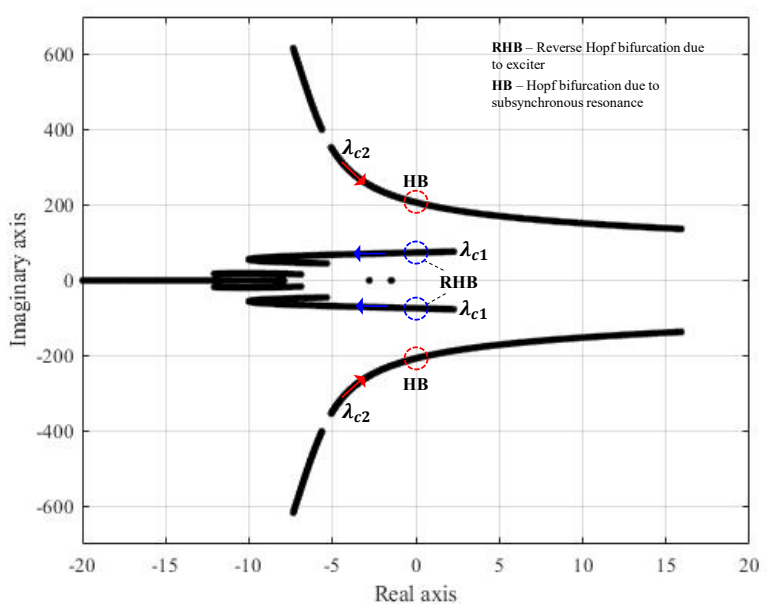

Figure 4: Eigenvalue trajectory for $K_{a}=2000$

$K_{c r}=56 \%$ with $\lambda_{c r}=0.0792 \pm j 189.43$.

\subsection{Influence of AVR parameters on stability domain}

The influence of exciter parameters on the stability domain of the system with respect to series compensation level is explored in this section through eigenvalue analysis. The parameters under study are the gain of the exciter and the time constants of the AVR and measurement transducer.

\subsubsection{Influence of exciter gain}

Eigenvalues of the system are computed for the following operating conditions: the induction motor slip is at $2 \%$ and the $X / R$ ratio of the line is assumed to be 25 , exciter of the DG has a gain and time constant of 2000 and $25 \mathrm{~ms}$ respectively and the measurement transducer time constant of the AVR of the DG is 10 ms. The eigenvalue trajectory of the system are presented in Fig. 3 as the degree of series compensation $K_{c}$ is varied from $1 \%$ to $98 \%$. The non-oscillatory modes are stable for the entire range of series compensation considered. When $K_{c}$ is at $1 \%$, there is an oscillatory mode on the right half of the complex 
Table 3: Eigenvalues for base case

\begin{tabular}{|c|c|c|}
\hline$\lambda_{i}$ & $-\sigma \pm j \omega$ & Dominant states \\
\hline$\lambda_{1}$ & $-40.188 \pm j 2086.8$ & $\Delta I_{d L}, \Delta I_{d L}, \Delta I_{q L}, \Delta I_{d s}, \Delta I_{q s}, \Delta I_{d r}, \Delta I_{q r}, \Delta V_{d m}, \Delta V_{q m}$ \\
\hline$\lambda_{2}$ & $-51.454 \pm j 1330$ & $\Delta I_{d L}, \Delta I_{d L}, \Delta I_{q L}, \Delta I_{d s}, \Delta I_{q s}, \Delta I_{d r}, \Delta I_{q r}, \Delta V_{d m}, \Delta V_{q m}$ \\
\hline$\lambda_{3}$ & $-7.4056 \pm j 557.92$ & $\Delta I_{d L}, \Delta I_{d L}, \Delta I_{q L}, \Delta I_{d s}, \Delta I_{q s}, \Delta I_{d r}, \Delta I_{q r}, \Delta V_{d c}, \Delta V_{q c}$ \\
\hline$\lambda_{4}$ & $-0.2358 \pm j 194.8$ & $\Delta I_{d L}, \Delta I_{d L}, \Delta I_{q L}, \Delta I_{d s}, \Delta I_{q s}, \Delta I_{d r}, \Delta I_{q r}, \Delta V_{d c}, \Delta V_{q c}$ \\
\hline$\lambda_{5}$ & $-7.1786 \pm j 28.759$ & $\Delta I_{d s}, \Delta I_{q s}, \Delta I_{d r}, \Delta I_{q r}, \Delta E_{q}^{\prime}, \Delta V_{x}, \Delta E_{f d}$ \\
\hline$\lambda_{6}$ & $-10.157 \pm j 20.166$ & $\Delta I_{d s}, \Delta I_{q s}, \Delta I_{d r}, \Delta I_{q r}, \Delta E_{q}^{\prime}, \Delta s_{m}$ \\
\hline$\lambda_{7}$ & -106.99 & $\Delta I_{d s}, \Delta I_{q s}, \Delta I_{d r}, \Delta I_{q r}, \Delta E_{q}^{\prime}, \Delta V_{x}, \Delta E_{f d}$ \\
\hline$\lambda_{8}$ & -24.485 & $\Delta I_{d s}, \Delta I_{q s}, \Delta I_{d r}, \Delta I_{q r}, \Delta E_{f d}$ \\
\hline$\lambda_{9}$ & -17.112 & $\Delta I_{d s}, \Delta I_{q s}, \Delta I_{d r}, \Delta I_{q r}, \Delta T_{m}, \Delta \omega$ \\
\hline$\lambda_{10}$ & -2.7822 & $\Delta T_{m}, \Delta \omega$ \\
\hline$\lambda_{11}$ & -1.4834 & $\Delta I_{d s}, \Delta I_{q s}, \Delta I_{d r}, \Delta I_{q r}, \Delta E_{d}^{\prime}$ \\
\hline
\end{tabular}

Table 4: Critical eigenvalues for different $\mathrm{X} / \mathrm{R}$ ratios

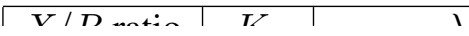

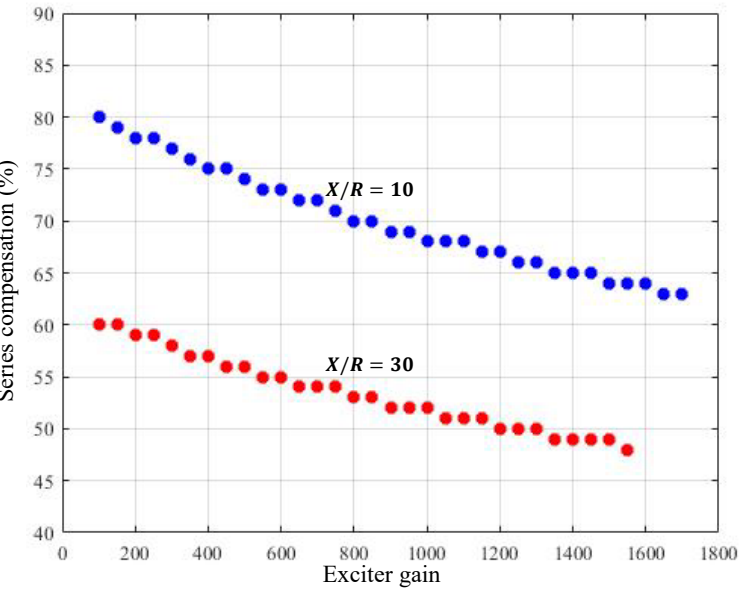

Figure 5: Hopf bifurcation points for varying exciter gains

plane $\left(\lambda_{c 1}=2.2759 \pm j 76.585\right)$ making the system unstable. As the compensation increases, $\lambda_{c 1}$ moves toward the left half of the complex plane and when $K_{c}$ exceeds $24 \%, \lambda_{c 1}$ moves into the left half of the complex plane which results in a stable system as a consequence of a reverse Hopf bifurcation. As $K_{c}$ further increases, the system becomes unstable once again as another oscillatory mode moves to the right half of the complex plane when $K_{c}$ exceeds $49 \%$. The critical eigenvalues at this point are $\lambda_{c 2}=0.1471 \pm j 205.38$. Instability in this case is a result of a Hopf bifurcation occurring due to SSR. Critical eigenvalues and participation factors of the system for 2 different series compensation levels of $15 \%$ and $50 \%$ are presented in Table 3.

The eigenvalues of the system were further computed for various exciter gains for $X / R$ ratios of 10 and 30. In Fig. 5, the variation of Hopf bifurcation points with respect to exciter gain and series compensation levels are shown. For an AVR gain of 100, the system with $X / R$ ratios of 10 and 30 loses stability through a Hopf bifurcation due to SSR when the series compensation level is $80 \%$ and $60 \%$ respectively. As the AVR gain increases, the percentage series compensation at which SSR occurs decreases resulting in smaller stability domains.

As the AVR gain was increased to 1600 for an $X / R$ ratio of 30 , the system was unstable for $K_{c}$ of $1 \%$ to $3 \%$ and when $K_{c}$ is increased beyond $4 \%$, the system becomes stable causing a reverse Hopf bifurcation. The system remains stable for values of $K_{c}$ up to $47 \%$ and as $K_{c} \geq 48 \%$, the system loses stability due to SSR. As the AVR gain is increased to 1800, the system experiences a reverse Hopf bifurcation (RHB) and a Hopf bifurcation (HB) at $K_{c}$ values of $16 \%$ and $47 \%$ respectively. Similar phenomenon was observed for the system with $X / R$ ratio of 10 , however the stability domains were found to be much higher. For example, for an AVR gain of 1800 in the system with $X / R=10$, the RHB and HB points correspond to $K_{c}$ values of $5 \%$ and $62 \%$ respectively. Hence, from this it can be inferred that for higher AVR gains, value of $K_{c}$ at which the reverse Hopf bifurcation occurs increases which further reduces the size of the stability 
Table 5: Critical eigenvalues for AVR gain of 2500

\begin{tabular}{|c|c|c|}
\hline$K_{c}$ & $\lambda_{c r}$ & Dominant states \\
\hline $15 \%$ & $0.9597 \pm j 75.013$ & $\Delta I_{d s}, \Delta I_{q s}, \Delta I_{d r}, \Delta I_{q r}, \Delta E_{q}^{\prime}, \Delta V_{x}, \Delta E_{f d}$ \\
\hline $50 \%$ & $0.094 \pm j 170.26$ & $\Delta I_{d L}, \Delta I_{q L}, \Delta I_{d s}, \Delta I_{q s}, \Delta I_{d r}, \Delta I_{q r}, \Delta V_{d c}, \Delta V_{q c}$ \\
\hline
\end{tabular}

domain. Overall, the size of the stability domain with respect to $K_{c}$ is much smaller for a weaker system especially for high AVR gains.

\subsection{Influence of exciter time constant}

The time constant of the AVR has a significant impact on the stability domain of the system with respect to series compensation which would be described in this section. Eigenvalues of the system were analyzed as the series compensation is varied from $1 \%$ to $98 \%$ for various values of exciter time constant. This was done for systems with $X / R$ ratios of 10 and 30 . This analysis was performed for AVR gains of 500 and 1000 and the results are presented in Tables 6 and 7 respectively. Stability domain of the system with respect to $K_{c}$ is increased as the AVR time constant is increased from $1 \mathrm{~ms}$ to $40 \mathrm{~ms}$ as the Hopf bifurcation point due to SSR is moved from $K_{c}=51 \%$ to $K_{c}=77 \%$ for the system with $X / R=10$. When the time constant is increased to $42 \mathrm{~ms}$, instability due to a Hopf bifurcation occurs due to a mode corresponding to the exciter at $K_{c}=75 \%$. As the time constant increases further to $70 \mathrm{~ms}$, the stability domain shrinks further as the Hopf bifurcation point due to the exciter is shifted to $K_{c}=6 \%$. When $T_{a} \geq 80 \mathrm{~ms}$, the stable operation of the system is not possible for any level of $K_{c}$ as the system is unstable for $K_{c}$ starting at $1 \%$.

The same phenomenon occurs for the system with $X / R=30$, however, the stability domain starts decreasing when $T_{a} \geq 59 \mathrm{~ms}$ which is higher compared to the previous case with $X / R=10$. Stable operation of the system is impossible for $T_{a} \geq 100 \mathrm{~ms}$ which is also higher compared to the previous case. Also, when the time constant $T_{a}=59 \mathrm{~ms}$, as $K_{c}$ changes from $58 \%$ to $59 \%, 2$ pairs of complex eigenvalues $\left(\lambda_{c 1}=0.0277 \pm j 184.7, \lambda_{c 2}=0.0194 \pm j 23.495\right.$ at $K_{c}=59 \%$ ) crossover from the left to the right half of the complex plane resulting in a Hopf-Hopf bifurcation.

The exciter gain is increased to 1000 and the same analysis was performed on the systems with $X / R$ ratios of 10 and 30 and the results are presented in Table 7. The stability domain with respect to $K_{c}$ is greater for the system with $X / R=10$ up to a value of $T_{a}$ close to $40 \mathrm{~ms}$ compared to the system with $X / R=30$. When $T_{a}=50 \mathrm{~ms}$, the system with $X / R=10$ has a much smaller stability domain as the system loses stability due to a Hopf bifurcation with the exciter mode causing instability at $K_{c}=36 \%$. This is much smaller compared to the Hopf bifurcation point which corresponds to $K_{c}=56 \%$ due to SSR for the system with $X / R=30$. Also note that as $T_{a}$ varies from 1 to $40 \mathrm{~ms}$, the stability domain increases as the critical value of $K_{c}$ causing a Hopf bifurcation due to SSR increases for each of the systems with $X / R$ ratios of 10 and 30 respectively. Furthermore, the system also undergoes a reverse Hopf bifurcation for $T_{a} \geq 64 \mathrm{~ms}$ at smaller values of $K_{c}$ in addition to the Hopf bifurcation which occurs due to SSR as $K_{c}$ is increased. This shows that for it may be impossible to operate a weak system in a stable manner without a minimum level of series compensation.

\subsection{Influence of time constant of measure- ment transducer}

Eigenvalue analysis was conducted for the system with $X / R$ ratios of 10 and 30 for various values of time constant of the measurement transducer. Just as it was described in the previous section with respect to $T_{a}$, here, the stability domain increases for both the systems with $X / R$ ratios of 10 and 30 as $T_{x}$ increases up to $20 \mathrm{~ms}$. Beyond that, as $T_{x} \geq 25$, the system with $X / R=10$ becomes unstable at lower values of $K_{c}$ due to the exciter mode. This phenomenon is observed in the system with $X / R=30$ as well, however, at a higher value of $T_{x}$ at $35 \mathrm{~ms}$. Hence, up to a certain value of $T_{x}$, the stability domain of the stronger system is bigger than that of the weaker system. However, beyond a certain value of $T_{x}$, the weaker system has a larger stability domain. As the AVR gain is increased to 1000 , the system shows similar behavior. In addition to Hopf bifurcations due to SSR and exciter mode destabilization, reverse Hopf bifurcations occur as well at small values of $K_{c}$. Hence, systems using AVRs with high gains need a minimum level of $K_{c}$ in order to have stable operation. Depending on the values of $T_{x}$ and $T_{a}$, the upper limit of $K_{c}$ is determined either by SSR or exciter mode destabilization.

\section{Time domain simulation results}

The nonlinear dynamic model of the system is programmed in SIMULINK and the results generated are 


\begin{tabular}{|c|c|c|c|c|}
\hline \multirow{2}{*}{$T_{a}(\mathrm{~ms})$} & \multicolumn{2}{|c|}{$X / R=10$} & \multicolumn{2}{c|}{$X / R=30$} \\
\cline { 2 - 5 } & $K_{c r}(\%)$ & $\lambda_{c r}=-\sigma \pm j \omega$ & $K_{c r}(\%)$ & $\lambda_{c r}=-\sigma \pm j \omega$ \\
\hline 1 & 51 & $0.0203 \pm j 224.79$ & 38 & $0.0609 \pm j 223.53$ \\
\hline 5 & 57 & $0.0423 \pm j 214.73$ & 43 & $0.0962 \pm j 212.61$ \\
\hline 10 & 65 & $0.0473 \pm j 203.49$ & 49 & $0.0689 \pm j 201.35$ \\
\hline 20 & 72 & $0.0279 \pm j 194.52$ & 54 & $0.0034 \pm j 192.75$ \\
\hline 30 & 75 & $0.027 \pm j 190.88$ & 57 & $0.0831 \pm j 187.81$ \\
\hline 40 & 77 & $0.0644 \pm j 188.5$ & 58 & $0.0547 \pm j 186.24$ \\
\hline 50 & 54 & $0.0019 \pm j 24.798$ & 59 & $0.0783 \pm j 184.65$ \\
\hline 60 & 30 & $0.0292 \pm j 23.87$ & 58 & $0.0428 \pm j 23.408$ \\
\hline 70 & 6 & $0.0012 \pm j 23.116$ & 45 & $0.0469 \pm j 22.703$ \\
\hline 80 & 1 & $0.4935 \pm j 22.09$ & 32 & $0.0189 \pm j 22.111$ \\
\hline
\end{tabular}

Table 6: Critical eigenvalues for various AVR time constants at an AVR gain of 500

\begin{tabular}{|c|c|c|c|c|}
\hline \multirow{2}{*}{$T_{a}(\mathrm{~ms})$} & \multicolumn{2}{|c|}{$X / R=10$} & \multicolumn{2}{c|}{$X / R=30$} \\
\cline { 2 - 5 } & $K_{c r}(\%)$ & $\lambda_{c r}=-\sigma \pm j \omega$ & $K_{c r}(\%)$ & $\lambda_{c r}=-\sigma \pm j \omega$ \\
\hline 1 & 37 & $0.1122 \pm j 247.35$ & 27 & $0.1024 \pm j 247.53$ \\
\hline 10 & 56 & $0.0463 \pm j 215.27$ & 42 & $0.0250 \pm j 213.76$ \\
\hline 20 & 66 & $0.0725 \pm j 201.77$ & 50 & $0.1073 \pm j 199.22$ \\
\hline 30 & 70 & $0.0172 \pm j 196.79$ & 53 & $0.0520 \pm j 194.2$ \\
\hline 40 & 73 & $0.0694 \pm j 193.13$ & 55 & $0.0609 \pm j 190.94$ \\
\hline 50 & 36 & $0.0147 \pm j 36.324$ & 56 & $0.032 \pm j 189.36$ \\
\hline
\end{tabular}

Table 7: Critical eigenvalues for various AVR time constants at an AVR gain of 1000

presented in this section. The responses of the system with respect to time are studied for AVR gain $K_{a}$ of 500 and different values of time constants of the AVR control system. The series compensation level for the system is at $50 \%$ which is realistic for a weak system with an $X / R$ ratio of 30 .

\subsection{Exciter time constant $T_{a}=10 \mathrm{~ms}$}

The responses of induction motor speed and terminal voltage are shown in Fig.6 for a disturbance in the form of a $20 \%$ increase in the load torque on the induction motor. In this case, the AVR limits have not been modeled as a result of which, oscillations in the voltage increase with time. This is a consequence of a subcritical Hopf bifurcation as a result of SSR which causes excessive voltages and currents.

Limits on the excitation system have been imposed and the simulations were repeated and the time responses are presented in Fig. 7. Exciter limits impose an upper and lower limit on the field voltage applied to the rotor winding of the synchronous machine resulting in an arrest on the growth of oscillations in the system states. The system now behaves as if the nature of the bifurcation is supercritical resulting in sustained oscillations of fixed amplitude with respect to time. Phase plane trajectory of the induction motor speed in Fig. 8 reveal an unstable system with growing oscillations if the AVR limits are neglected and a system with oscillations bounded in magnitude if the AVR limits are taken into account.

\subsection{Exciter time constant $T_{a}=70 \mathrm{~ms}$}

Time traces of induction motor speed and terminal voltage are presented in Fig. 9 when the AVR limits of the synchronous machine are neglected. The system shows an oscillatory response bounded in magnitude characterizing a supercritical Hopf bifurcation. The amplitude of oscillations appear to continually change within a bound for the quantities presented. However, with the AVR limits included, the oscillations appear to be bounded with fixed amplitude characterizing a supercritical bifurcation in this case as well. These are evident from the time responses of induction motor speed and terminal voltage as shown in Fig. 10.

Phase-plane trajectories of induction motor speed when the AVR time constant is set at $70 \mathrm{~ms}$ are presented in Fig. 11. In the case where the AVR limits are neglected, a supercritical Hopf bifurcation is observed in the system which appears to undergo repeated period doublings. This is evident in the one to the left 


\begin{tabular}{|c|c|c|c|c|}
\hline \multirow{2}{*}{$T_{x}(\mathrm{~ms})$} & \multicolumn{2}{|c|}{$X / R=10$} & \multicolumn{2}{c|}{$X / R=30$} \\
\cline { 2 - 5 } & $K_{c r}(\%)$ & $\lambda_{c r}=-\sigma \pm j \omega$ & $K_{c r}(\%)$ & $\lambda_{c r}=-\sigma \pm j \omega$ \\
\hline 1 & 64 & $0.052 \pm j 205.81$ & 48 & $0.0699 \pm j 204$ \\
\hline 5 & 69 & $0.0862 \pm j 198.52$ & 52 & $0.0973 \pm j 196.32$ \\
\hline 10 & 74 & $0.0522 \pm j 192.07$ & 56 & $0.0792 \pm j 189.43$ \\
\hline 15 & 77 & $0.0866 \pm j 188.41$ & 58 & $0.0713 \pm j 186.17$ \\
\hline 20 & 78 & $0.0426 \pm j 187.26$ & 59 & $0.0583 \pm j 184.59$ \\
\hline 25 & 56 & $0.014 \pm j 26.955$ & 60 & $0.0919 \pm j 183.01$ \\
\hline 30 & 30 & $0.0039 \pm j 26.951$ & 60 & $0.0459 \pm j 183.06$ \\
\hline 35 & 1 & $0.0933 \pm j 27.902$ & 47 & $0.0412 \pm j 26.127$ \\
\hline
\end{tabular}

Table 8: Critical eigenvalues for various measurement time constants at an AVR gain of 500

\begin{tabular}{|c|c|c|c|c|}
\hline \multirow{2}{*}{$T_{x}(\mathrm{~ms})$} & \multicolumn{2}{|c|}{$X / R=10$} & \multicolumn{2}{c|}{$X / R=30$} \\
\cline { 2 - 5 } & $K_{c r}(\%)$ & $\lambda_{c r}=-\sigma \pm j \omega$ & $K_{c r}(\%)$ & $\lambda_{c r}=-\sigma \pm j \omega$ \\
\hline 1 & 53 & $0.0661 \pm j 221.22$ & 39 & $0.004 \pm j 221$ \\
\hline 5 & 61 & $0.1190 \pm j 208.75$ & 46 & $0.0354 \pm j 206.65$ \\
\hline 10 & 68 & $0.0008 \pm j 199.28$ & 52 & $0.1094 \pm j 195.83$ \\
\hline 15 & 73 & $0.1027 \pm j 192.97$ & 55 & $0.084 \pm j 190.8$ \\
\hline
\end{tabular}

Table 9: Critical eigenvalues for various measurement time constants at an AVR gain of 1000

in Fig. 11. The amplitude of oscillations although bounded appear to change continuously in a random manner which is the characteristic of 'quasi-chaos'. However, with the AVR limits modeled, the phaseplane trajectory of induction motor speed shows a well-defined limit cycle and the absence of a period doubling bifurcation. This is due to the fact that with AVR limits modeled, the voltage applied to the field winding is allowed to vary only between \pm 3 pu. This limit is much lower than the minimum amplitude of oscillations in $E_{f d}$ when it varies in a random manner when AVR limits are neglected. This imposes a bound on the variations in the generator terminal voltage which is reflected at the motor terminal voltage and impacts the speed of the motor which varies only within a limit.

Further investigations were carried out with regards to the period-doubling bifurcation observed in the system when AVR limits were neglected. The system was subject to different step increases in the induction motor load torque and phase-plane trajectories of the induction motor speed are plotted and are shown in Fig. 12. With the system subject to a $25 \%$ increase in the load torque, the number of period doublings are hard to count as the trajectories are so close to each other. As the step change was increased to $30 \%, 2$ period doublings can be observed. The number of period doublings reduced to 1 when the load torque disturbance was increased to $35 \%$ and finally, the period doubling bifurcation is found to be absent when the load torque disturbance magnitude was increased to $45 \%$.

\section{Conclusions}

Influence of generator AVR parameters on the stability domain of the system with respect to series compensation has been studied in this paper and the following conclusions are drawn:

- The gain of the AVR determines the critical series compensation level at which SSR occurs. If an AVR of large gain is used, the system tends to lose stabilty due to SSR at a lower value of series compensation. Furthermore, large AVR gains also lead to a reverse Hopf bifurcation for a certain level of series compensation which shows that a certain minimum level of series compensation is required for stable operation.

- Time constants of the AVR and the voltage measurement transducer are critical in determining the stability domain of the system with respect to series compensation. The upper limit of series compensation is fixed by the Hopf bifurcation point at which either the subsynchronous resonant mode or the exciter mode becomes destabilized.

- The upper limit of series compensation is determined by the series compensation level at 


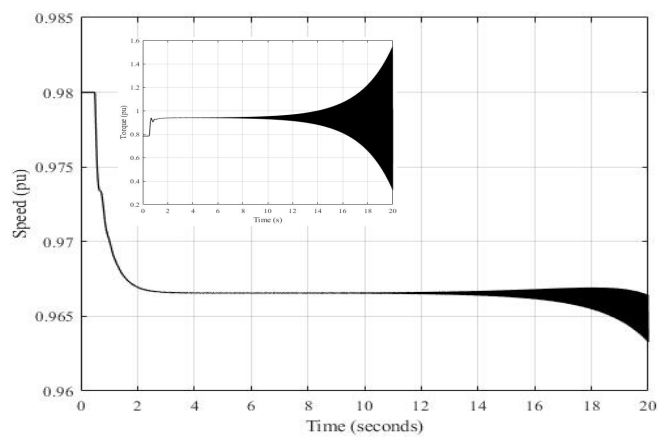

(a) Induction motor speed

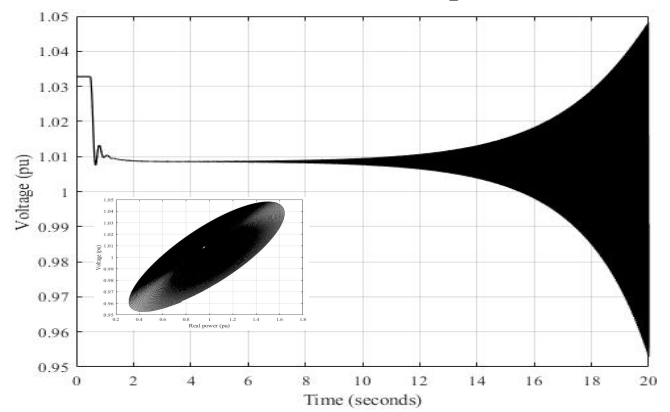

(b) Motor terminal voltage

Figure 6: Time responses for the system without AVR limits

which SSR occurs for a system with a fast acting AVRs and measurement transducers. On the other hand, in systems consisting of exciters with larger time constants, the upper limit is determined by the series compensation level at which the low frequency mode related to the excitation system loses stability.

- Stability domain of the relatively stronger system is larger if the upper limit of series compensation is determined by the level at which SSR occurs. However, the weaker system has a larger stability domain if the upper limit of series compensation is determined by the critical level at which the exciter mode becomes unstable due to a Hopf bifurcation.

- Depending upon magnitude of disturbance applied at certain critical values of AVR parameters and series compensation, the limit cycle generated as a result of the Hopf bifurcation may undergo repeated bifurcations resulting in multiple periodic orbits culminating in a form of chaos.

- There is a possibility of a Hopf-Hopf bifurcation in the system for a certain combination of percentage series compensation and AVR time constant. However, the occurrence of this maybe only on a case-by-case basis.

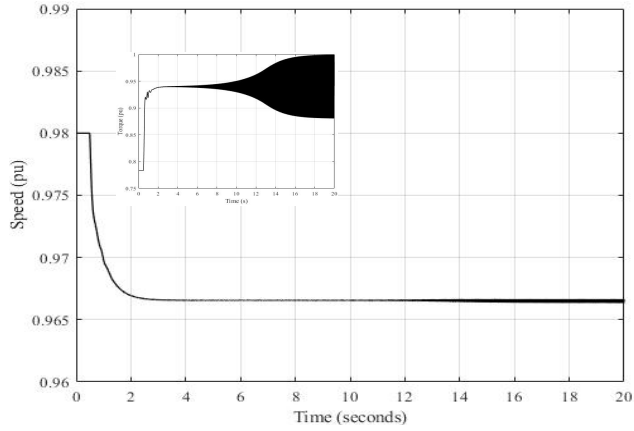

(a) Induction motor speed

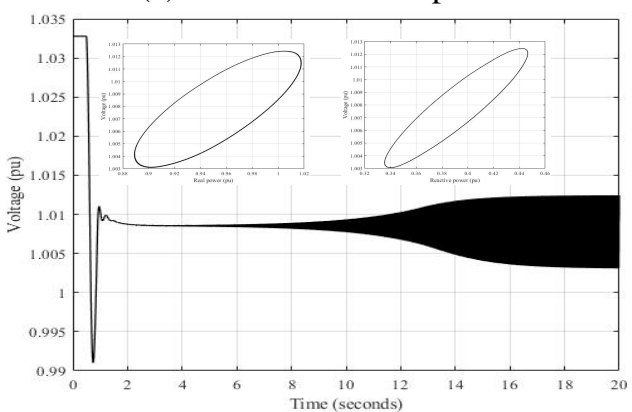

(b) Motor terminal voltage

Figure 7: Time responses for the system with AVR limits

- This study emphasises the importance of properly selecting the parameters for the AVR of the synchronous generator as bifurcations occur in the induction motor operation when parameters crosses certain limits especially in series compensated systems. As weak grids become more common with decentralized generation, microgrids and isolated networks and with the widespread use of induction motors as loads, the importance of understanding and predicting dynamic responses is vital to achieve safer, more secure and more reliable operation.

Acknowledgements: The authors would like to thank the funders: Oklahoma Center for the Advancement of Science and Technology (project no. AR18-073) and the Oklahoma Gas \& Electric Company (project no. A18-0274).

\section{References:}

[1] Reader's guide to subsynchronous resonance. IEEE Transactions on Power Systems, 7(1):150 157, 1992.

[2] P. Kundur. Power system stability. Power system stability and control, pages 7-1, 2007. 

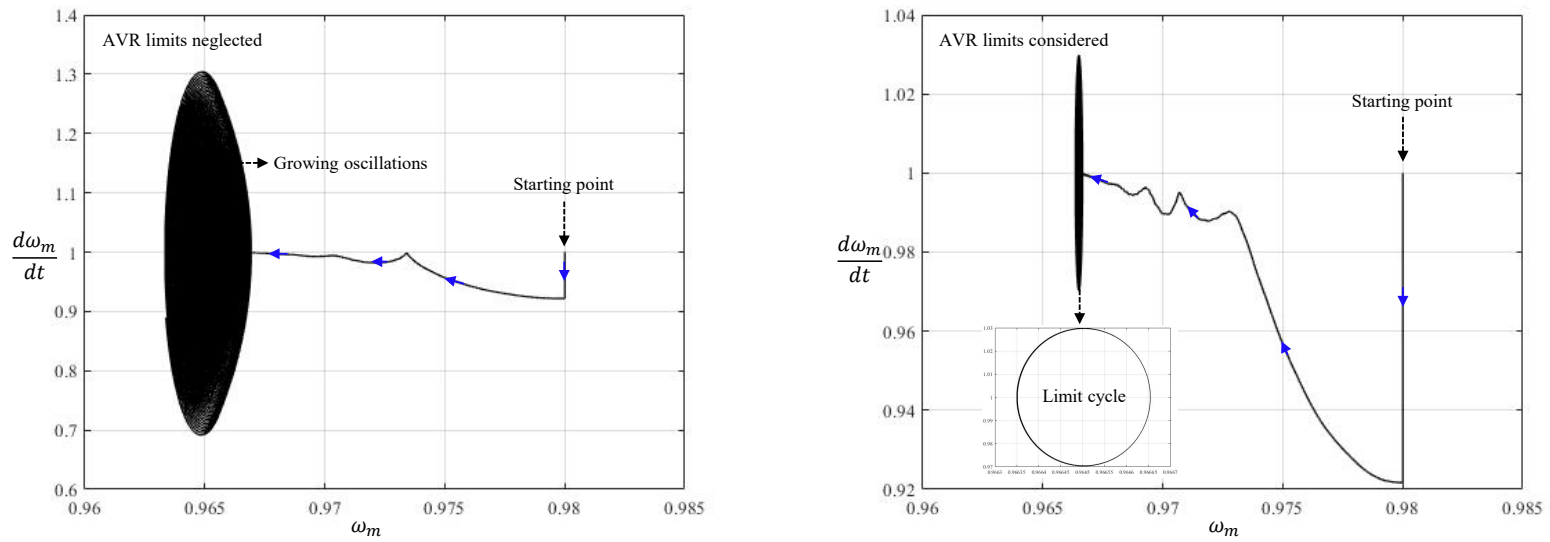

Figure 8: Phase plane trajectory of induction motor speed for $T_{a}=10 \mathrm{~ms}$

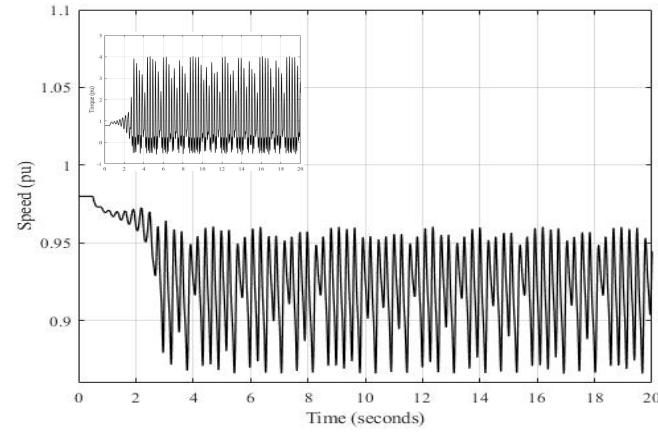

(a) Induction motor speed

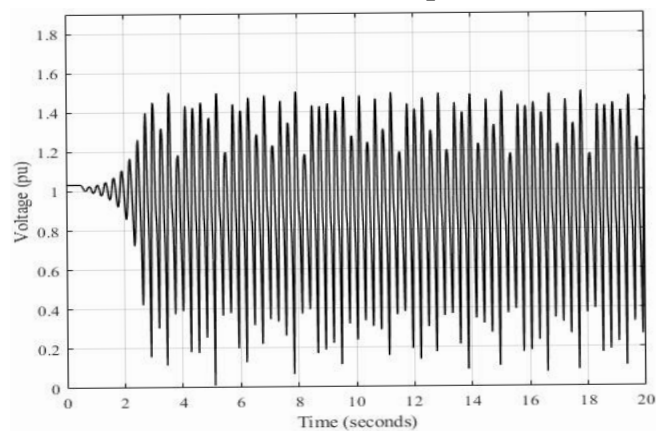

(b) Motor terminal voltage

Figure 9: Time responses for the system without AVR limits

[3] K.R. Padiyar. Analysis of subsynchronous resonance in power systems. Springer Science \& Business Media, 2012.

[4] C. He, D. Sun, L. Song, and L. Ma. Analysis of subsynchronous resonance characteristics and influence factors in a series compensated transmission system. Energies, 12(17):3282, 2019.

[5] Md A. Mahmud, M.J. Hossain, and H.R. Pota. Effects of large dynamic loads on power sys-

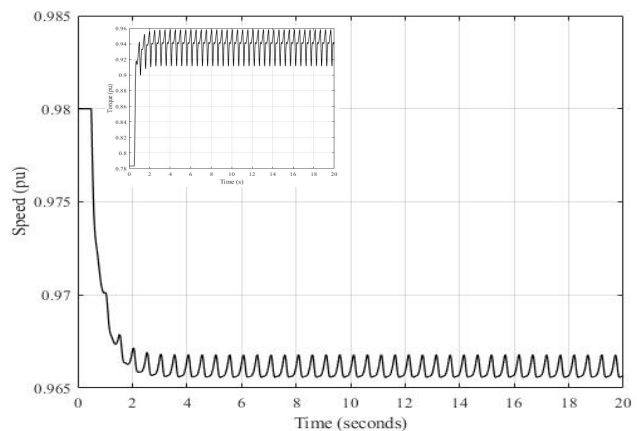

(a) Induction motor speed

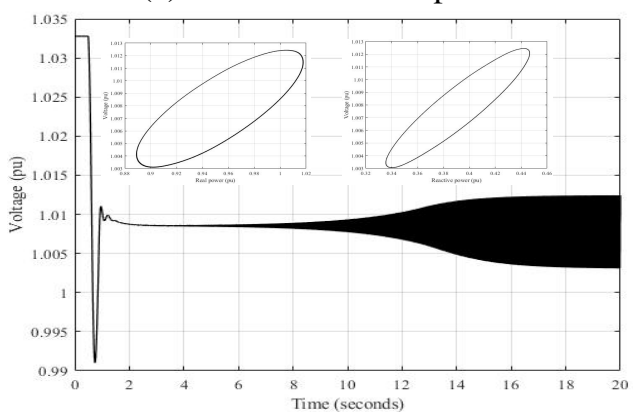

(b) Motor terminal voltage

Figure 10: Time responses for the system with AVR limits

tem stability. International Journal of Electrical Power \& Energy Systems, 44(1):357-363, 2013.

[6] A.H. Nayfeh, A. Harb, C. Chin, A.M.A. Hamdan, and L. Mili. A bifurcation analysis of subsynchronous oscillations in power systems. Electric power systems research, 47(1):21-28, 1998.

[7] A.H. Nayfeh, A. Harb, C. Chin, A.M.A. Hamdan, and L. Mili. Application of bifurcation theory to subsynchronous resonance in power sys- 

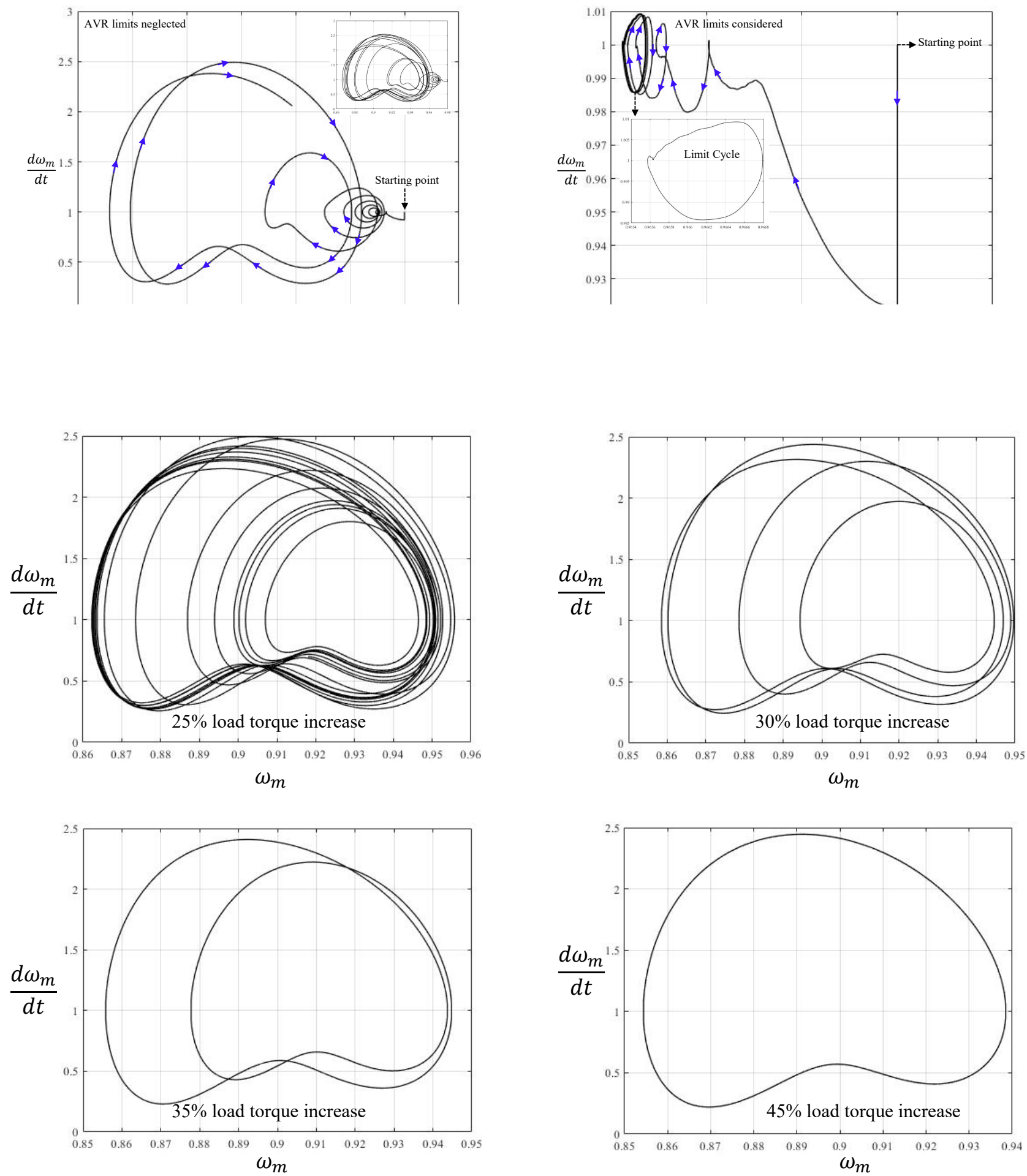

Figure 12: Period doublings for various step changes in load torque

tems. International Journal of Bifurcation and Chaos, 8(01):157-172, 1998.

[8] M. Varghese, F.F. Wu, and P. Varaiya. Bifurcations associated with sub-synchronous resonance. IEEE Transactions on Power Systems, 13(1):139-144, 1998.
[9] W. Zhu, R.R. Mohler, R. Spee, W.A. Mittelstadt, and D. Maratukulam. Hopf bifurcations in a SMIB power system with SSR. IEEE Transactions on Power Systems, 11(3):1579-1584, 1996.

[10] M.S. Widyan. On the effect of AVR gain 
on bifurcations of subsynchronous resonance in power systems. International Journal of Electrical Power \& Energy Systems, 32(6):656-663, 2010.

[11] W. Ji and V. Venkatasubramanian. Hard-limit induced chaos in a fundamental power system model. International Journal of Electrical Power \& Energy Systems, 18(5):279-295, 1996.

[12] B.C. Rout, D.K. Lal, and A.K. Barisal. Control of period doubling bifurcation in an SMIB power system using adaptive controller based on lasalle's invariant principle. Cogent Engineering, 4(1):1362804, 2017.

[13] P.S. Moses and M.A.S. Masoum. Modeling subharmonic and chaotic ferroresonance with transformer core model including magnetic hysteresis effects. WSEAS Transactions on Power Systems, 2009.

[14] P.S. Moses, M.A.S. Masoum, and H.A. Toliyat. Impacts of hysteresis and magnetic couplings on the stability domain of ferroresonance in asymmetric three-phase three-leg transformers. IEEE Transactions on Energy Conversion, 26(2):581592, 2010.

[15] A.A. Mahmoud, T.H. Ortmeyer, R.G. Harley, and C. Calabrese. Effects of reactive compensation on induction motor dynamic performance. IEEE Transactions on Power Apparatus and Systems, (3):841-846, 1980.

[16] D.J.N. Limebeer and R.G. Harley. Subsynchronous resonance of single-cage induction motors. In IEE Proceedings B-Electric Power Applications, volume 128, pages 33-42. IET, 1981.

[17] O. Ojo. Electro-mechanical subsynchronous resonance of a series capacitive compensated line start induction motor drive. In Conference Record of the IEEE Industry Applications Society Annual Meeting, pages 238-247. IEEE, 1989.

[18] P.W. Sauer, M.A. Pai, and J.H. Chow. Power system dynamics and stability: with synchrophasor measurement and power system toolbox. John Wiley \& Sons, 2021.

[19] P.C. Krause, O. Wasynczuk, S.D. Sudhoff, and S.D. Pekarek. Analysis of electric machinery and drive systems, volume 75 . John Wiley \& Sons, 2013.
Contribution of Individual Authors to the Creation of a Scientific Article (Ghostwriting Policy)

The authors equally contributed in the present research, at all stages from the formulation of the problem to the final findings and solution.

\section{Sources of Funding for Research Presented in a} Scientific Article or Scientific Article Itself

No funding was received for conducting this study.

\section{Conflict of Interest}

The authors have no conflicts of interest to declare that are relevant to the content of this article.

Creative Commons Attribution License 4.0 (Attribution 4.0 International, CC BY 4.0)

This article is published under the terms of the Creative Commons Attribution License 4.0 https://creativecommons.org/licenses/by/4.0/deed.en 